\title{
IN ELDERLY PATIENTS WITH NON-ST-SEGMENT ELEVATION ACUTE CORONARY SYNDROME
}

doi:10.1136/heartjnl-2012-302920k.6

${ }^{1}$ Wang Xue-zhong, ${ }^{1}$ Wang Yue-song, ${ }^{1}$ Wang Shao-jun, ${ }^{1}$ Fan Oin, ${ }^{2}$ Yan Zhi-jian,

${ }^{2}$ Yan Zhi-jian. ${ }^{1}$ Department of Cardiology, Manshan People's Hosptial, Manshan, Anhui 243000, China; ${ }^{2}$ Department of Cardiology, the First Affiliated Hospital, Nanjing Medical University, Nanjing 210029, China

Objectives This study was designed to evaluate the peri-procedural myocardial injury and safety profile of atorvastatin in patients over 70 years old with non-ST segment elevation acute coronary syndromes (NSTEACS).

Methods A total of 157 patients over 70 years old with NSTE-acute coronary syndromes were randomly divided into the pretreatment with loading dose atorvastatin group or control atorvastatin group. The serum levels of Creatine kinase-MB (CK-MB) and cardiac troponin I ( $\mathrm{cTnI}$ ) were measured at the baseline and at 8, 12 and $24 \mathrm{~h}$ after the procedure. Clinical outcome was measured by taking the main end points of major adverse cardiac events including cardiac death, nonfatal acute myocardial infarction, or revascularisation during 30 days follow-up.

Results he proportion of patients with the serum levels of CK-MB and cTnI above normal up-limit value and that with PCI-related myocardial infarction at 24-h was significantly lower in high dose atorvastatin group than than those in the control dose group ( $(37.9 \%$ vs $50.5 \%, p=0.002,5.4 \%$ vs $22.6 \%, p=0.041 ; 12.6 \%$ vs $33.4 \%, p=0.035,4.3 \%$ vs $11.8 \%, p=0.034)$.At multivariable analysis, pre-treatment with atoravastatin conferred an $67 \%$ risk reduction of PCI-related myocardial infarction. $(p<0.05$ No deaths and revascularisation were recorded at 30-day follow-up in both groups. Alanine aminotransferase has no difference in two groups.

Conclusions Short-term pretreatment with a high dose of atorvastatin significantly reduces PCI-related myocardial infarction in elderly patients with NSTEACS and enjoys similar safety profile. 